\title{
Preliminary results on approximating a wavefunction as an unconstrained sum of Slater determinants
}

\author{
Gregory Beylkin ${ }^{1, *}$, Martin J. Mohlenkamp ${ }^{2}$, and Fernando Pérez ${ }^{1}$ \\ ${ }^{1}$ Department of Applied Mathematics, University of Colorado at Boulder, 526 UCB, Boulder, CO 80309-0526, USA. \\ ${ }^{2}$ Department of Mathematics, Ohio University, 321 Morton Hall, Athens OH 45701, USA.
}

\begin{abstract}
A multiparticle wavefunction, which is a solution of the multiparticle Schrödinger equation, satisfies the antisymmetry condition, thus making it natural to approximate it as a sum of Slater determinants. Many current methods do so but, in addition, they impose structural constraints on the Slater determinants, such as orthogonality between orbitals or a particular excitation pattern. By removing these constraints, we hope to obtain much more efficient expansions.

We use an integral formulation of the problem, a Green's function iteration, and a fitting procedure based on the computational paradigm of separated representations. For constructing and solving a matrix-integral system of equations derived from antisymmetric inner products, we develop new algorithms with computational complexity competitive with current methods.

We describe preliminary numerical results and make some observations.
\end{abstract}

() 2007 WILEY-VCH Verlag GmbH \& Co. KGaA, Weinheim

Given the difficulties of solving the multiparticle Schrödinger equation, current numerical methods in quantum chemistry/physics are remarkably successful. Part of their success comes from efficiencies gained by imposing structural constraints on the wavefunction to match physical intuition. However, such methods scale poorly to high accuracy, and are biased to only reveal structures that were part of their own construction. In [3] we develop a method that allows better scaling to high accuracy and an unbiased exploration of the structure of the wavefunction by approximating it as an unconstrained sum of Slater determinants.

Motivated by the physical intuition that electrons may be excited into higher energy states, the Configuration Interaction (CI) family of methods choose a set of determinants with predetermined orbitals, and then optimize the coefficients used to combine them. When it is found insufficient, methods to optimize the orbitals, work with multiple reference states, etc., are introduced. A common feature of all these methods is that they impose some structural constraints on the Slater determinants, such as orthogonality of orbitals or an excitation pattern. As the requested accuracy increases, these structural constraints trigger an explosion in the number of determinants used, making the computation intractable for high accuracy. The a priori structural constraints present in CI-like methods also force the wavefunction to comply with such structure, whether or not it really is the case. For example, if you use a method that approximates the wavefunction as a linear combination of a reference state and excited states, you could not learn that the wavefunction is better approximated as a linear combination of several non-orthogonal, near-reference states. Thus, the choice of numerical method is not just a computational issue; it can help or hinder our understanding of the wavefunction.

Our goal is to construct an adaptive numerical method without imposing a priori structural constraints besides that of antisymmetry. In [3] we derive and present an algorithm for approximating a wavefunction with an unconstrained sum of Slater determinants, with fully-adaptive single-electron functions. In particular, we discard the notions of reference state and excitation of orbitals. The functions comprising the Slater determinants need not come from a particular basis set, be orthogonal, or follow some excitation pattern. They are computed so as to optimize the overall representation. In this respect we follow the philosophy of separated representations $[1,2]$, which allow surprisingly accurate expansions with remarkably few terms.

Our construction generates a solution using an iterative procedure based on nonlinear approximations via separated representations. We derive a system of integral equations that describe the fully-correlated many-particle problem. The computational core of the method is the repeated construction and solution of a matrix-integral system of equations.

Specifically, the following are distinctive features of our approach. We use:

- an adaptive representation for single-electron functions (our method does not depend on its particular details).

- an integral formulation of the multiparticle Schrödinger equation and a Green's function iteration to converge to the ground-state wavefunction. The Green's function is approximated by a sum of Gaussians.

- a variant of the so-called alternating least squares algorithm to reduce the error of our approximation using a sum of a given number of Slater determinants.

- antisymmetric inner products reduced to formulas involving only combinations of standard integrals. In particular, we avoid the direct application of the electron-electron potential and instead compute convolutions with the Poisson kernel.

* Corresponding author E-mail: Gregory.Beylkin@ colorado.edu, Phone: +01 3034926935 , Fax: +01 3034924066 
The Hamiltonian is the sum of kinetic, nuclear and electron-electron potential operators,

$$
\mathcal{H}=\mathcal{T}+\mathcal{V}+\mathcal{W}
$$

where the kinetic energy operator is $\mathcal{T}=-\frac{1}{2} \sum_{i=1}^{N} \Delta_{i}$ and $\Delta_{i}$ is the Laplacian for particle with index $i$, the nuclear potential for a set of nuclei at positions $\mathbf{R}_{\mathbf{a}}$ with charges $z_{a}$ is the multiplication operator $\mathcal{V}=\sum_{i=1}^{N} V\left(\mathbf{r}_{\mathbf{i}}\right)$, with $V(\mathbf{r})=$ $\sum_{a}-z_{a} /\left\|\mathbf{r}-\mathbf{R}_{\mathbf{a}}\right\|$, and the electron-electron interaction operator is the multiplication operator $\mathcal{W}=\frac{1}{2} \sum_{i=1}^{N} \sum_{j \neq i} 1 /\left\|\mathbf{r}_{\mathbf{i}}-\mathbf{r}_{\mathbf{j}}\right\|$. Our goal is to find negative discrete eigenvalues of the Hamiltonian,

$$
\mathcal{H} \psi=E \psi,
$$

providing energy levels of the system. We define the Green's function $\mathcal{G}_{\mu}=(\mathcal{T}-\mu \mathcal{I})^{-1}, \mu<0$, and use an integral formulation that eliminates the continuous spectrum,

$$
\psi=-(\mathcal{T}-E \mathcal{I})^{-1}(\mathcal{V}+\mathcal{W}) \psi
$$

The wavefunction is constructed via iteration

$$
\begin{aligned}
\tilde{\psi}_{n} & =-\mathcal{G}_{\mu_{n}}\left[(\mathcal{V}+\mathcal{W}) \psi_{n}\right] \\
\mu_{n+1} & =\mu_{n}-\left\langle(\mathcal{V}+\mathcal{W}) \psi_{n}, \psi_{n}-\tilde{\psi}_{n}\right\rangle /\left\|\tilde{\psi}_{n}\right\|^{2} \\
\psi_{n+1} & =\tilde{\psi}_{n} /\left\|\tilde{\psi}_{n}\right\|,
\end{aligned}
$$

where $\mu_{n} \longrightarrow E$. Since $\tilde{\psi}_{n}=-\mathcal{G}_{\mu_{n}}\left[(\mathcal{V}+\mathcal{W}) \psi_{n}\right]$ does not preserve the separated representation, we define $\tilde{\psi}_{n}$ to be the function of the correct separated form that minimizes

$$
\left\|\tilde{\psi}_{n}-\left(-\mathcal{G}_{\mu_{n}}\left[(\mathcal{V}+\mathcal{W}) \psi_{n}\right]\right)\right\|
$$

where we use the pseudo-norm induced by the usual Slater antisymmetrizer in order to assure convergence to an antisymmetric solution. This makes non-antisymmetric subspaces "invisible".

Among several devices to achieve appropriate complexity, we note a representation for the $N$-particle Green's function. We prove

Theorem For any $\epsilon>0, \mu<0$ and $N$, the $N$-particle Green's function $\mathcal{G}_{\mu}=\left(-\frac{1}{2} \sum_{i=1}^{N} \Delta_{i}-\mu \mathcal{I}\right)^{-1}$ has a separated representation with the relative error $\epsilon$ in the operator norm and with the number of terms, $L=\mathcal{O}\left(\left(\log \epsilon^{-1}\right)^{2}\right)$, independent of $\mu$ and $N$.

Preliminary numerical results demonstrate that our method recovers known electron configurations correctly (see e.g. correlation energy fraction for the Helium as a function of the separation rank). Further work is directed toward demonstrating advantages of our approach.

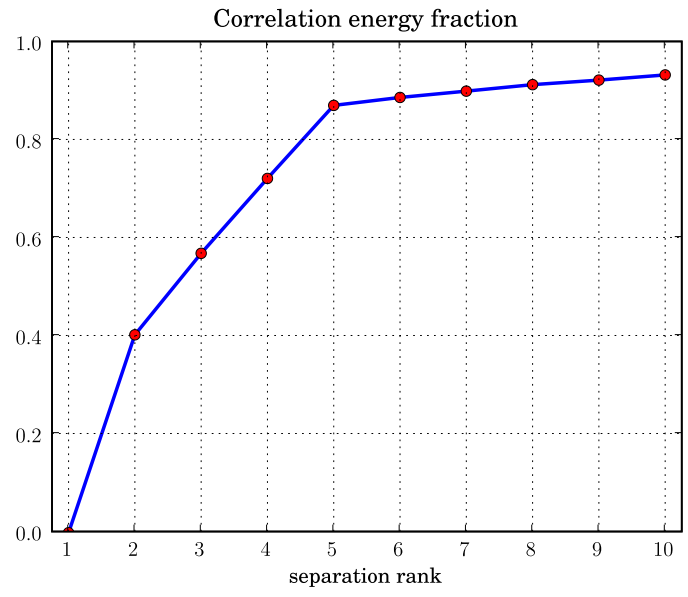

\section{References}

[1] G. Beylkin and M. J. Mohlenkamp. Numerical operator calculus in higher dimensions. Proc. Natl. Acad. Sci. USA, 99(16), 10246-10251 (2002).

[2] G. Beylkin and M. J. Mohlenkamp. Algorithms for numerical analysis in high dimensions. SIAM J. Sci. Comput., 26(6), 2133-2159 (2005).

[3] G. Beylkin, M. J. Mohlenkamp, and F. Pérez. Approximating a wavefunction as an unconstrained sum of Slater determinants. APPM Preprint \#554. Submitted J. Math. Phys., July 2007. 